\title{
Bifurcation Analysis and Chaos Control of a Fractional Order Portal Frame with Nonideal Loading Using Adaptive Sliding Mode Control
}

\author{
Karthikeyan Rajagopal, Anitha Karthikeyan, and Prakash Duraisamy \\ Center for Nonlinear Dynamics, Defence University, Bishoftu, Ethiopia \\ Correspondence should be addressed to Karthikeyan Rajagopal; rkarthiekeyan@gmail.com
}

Received 6 March 2017; Revised 11 April 2017; Accepted 3 May 2017; Published 20 July 2017

Academic Editor: Angelo Marcelo Tusset

Copyright (C) 2017 Karthikeyan Rajagopal et al. This is an open access article distributed under the Creative Commons Attribution License, which permits unrestricted use, distribution, and reproduction in any medium, provided the original work is properly cited.

\begin{abstract}
We investigate the chaotic oscillations in a fractional order model of a portal frame with nonideal loading. The bifurcation of the fractional order portal frame system for parameters and fractional orders are investigated. Bicoherence analysis shows the existence of quadratic nonlinearities. Fractional order adaptive sliding mode controllers are designed to suppress the chaotic oscillations with uncertain parameters. Power efficiency analysis of the FPGA implemented control scheme shows the maximum power utilization in the fractional order showing the largest Lyapunov exponent.
\end{abstract}

\section{Introduction}

Many recent researches have been dealing with the study of nonideal vibration systems when the excitation has an influence from the system response. These kind of nonideal vibrations systems are considered as major challenge in engineering research $[1,2]$. Chaotic vibrations of a portal frame system with nonideal loading and its control are presented by Tusset et al. [3]. Early researches on nonlinear vibration of frames under support motion do not consider the nonlinear elastic forces [4]. After two decades Brasil and Mazzilli studied the framed machine foundation considering both initial and elastic nonlinear effects including stiffness of columns and elastic deformations of the frames [5]. A simple portal frame structure with its nonlinear behavior under internal resonance conditions is analyzed for several energy levels [6].

Optimal linear feedback control for suppression chaotic oscillations was proposed by Rafikov et al., [7]. Nonlinear oscillations of a portal frame excited by a nonideal motor with limited power output are considered and, with slow increase of power levels, the possibilities of occurrence of Sommerfeld effect are investigated [8]. Dynamical behavior of an elastic nonideal portal frame with fractional nonlinearities taken into account the full interactions of the vibration systems with an energy source of limited supply is investigated [9]. A nonlinear control method based on the saturation phenomenon of systems coupled with quadratic nonlinearities in a shear building portal frame is studied [10]. A portal frame structure based on energy harvesters with piezo electric coupling exhibiting chaotic behavior is investigated and an optimal control scheme to regulate the energy captured to a designed operating frequency is preferred [11].

Recently many researchers have discussed fractional order calculus and its applications [12-14]. Fractional order nonlinear systems with different control approaches are investigated [15-17]. Numerical analysis and methods for simulating fractional order nonlinear system are proposed by Petras [18] and MATLAB solutions for fractional order chaotic systems, discussed by Trzaska Zdzislaw [19]. A fractional order control based approach is proposed for piezoactuated nanopositioning stage to suppress the vibration of the low-damped resonant mode and also to minimize the tracking error for nanopositioning applications and it is proposed [20]. A fractional order proportional-integral (FOPI) controller for a mass-spring-damper system which 
is poorly damped is proposed and it is proved that FOPI controllers are effective compared to the classical PI controllers [21]. A fractional order (FO) controller is proposed for solving the vibration suppression problem in civil structures experimented on a laboratory scaled steel structure, with one floor, modeled as a single degree-of-freedom system proposed and investigated [22]. The comparisons of the step responses of the integer order and the three types of fractional order damping systems are studied and it is shown that the optimal fractional order damping systems achieve much better step responses than optimal integer order systems [23]. The stability of fractional order systems using Lyapunov stability theory has been investigated in the literature [24, 25]. A fractional order controller to stabilize the unstable fixed points of an unstable open-loop system was proposed by Tavazoei and Haeri [26]. A bifurcation diagram shows the long term qualitative changes (equilibria or periodic orbits) of a system as a function of a bifurcation parameters of the system. The complete dynamics of the system with the variation of the parameters can be studied with the help of bifurcation diagram [27-29]. Nonlinear dynamical system undergoes abrupt qualitative changes when crossing bifurcation points [30]. For a more exhaustive qualitative analysis of a nonlinear dynamic system, it is compulsory to identify both singularities of the parameter plane and singularities of the phase plane [31].

Implementation of chaotic and hyperchaotic system using Field Programmable Gate Arrays (FPGA) is widely investigated [32-34]. Chaotic random number generators are implemented in FPGA for applications in image cryptography [35]. FPGA implemented Duffing oscillator based signal detectors are proposed by Rashtchi and Nourazar [36]. Digital implementation of chaotic multiscroll attractors is extensively investigated [32, 37]. Memristor based chaotic system and its FPGA circuits are proposed by Xu et al. [38]. A FPGA implementation of fractional order chaotic system using approximation method is investigated by Rajagopal et al. $[39,40]$.

Motivated by the above, in this paper we investigate the fractional order model of a portal frame (FOPF) under a nonideal excitation. Bifurcation plots of the FOPF system are investigated. To study the quadratic nonlinearities in the FOPF system, bicoherence contours along with the higher dimension power spectrum are presented. An adaptive sliding mode controller is designed to suppress chaotic oscillations and finally the proposed controller is implemented in FPGA for real-time implementation.

\section{Preliminaries and Problem Formulation}

We consider the horizontal motion of a portal frame with nonideal excitation [3] as described in Figure 1(a). The portal frame shown in Figure 1(a) is approximated with coupled oscillators [3] as shown in Figure 1(b).

As described in [3], the parameters of this dynamical system consist of $m_{0}, m_{1}$, the mass and unbalanced mass, $k_{1}, k_{\mathrm{nl}}$, linear and nonlinear stiffness, $c_{1}$, linear damping, $x_{1}, \varphi$, displacement and angular displacement, $J$, inertial moment, and $r$, eccentricity of the unbalanced mass. $d$ is related to the voltage applied across the armature of the DC motor and $s$ is a constant for each model of the DC motor considered. The resulting mathematical model of the structure is given by

$$
\begin{aligned}
& \left(m_{1}+m_{0}\right) \ddot{x}+b \dot{x}-k_{1} x+k_{\mathrm{nl}} x^{3} \\
& \quad=m_{0} r\left(\ddot{\varphi} \sin \varphi+\dot{\varphi}^{2} \cos \varphi\right), \\
& \left(J+r^{2} m_{0}\right) \ddot{\varphi}-r m_{0} \ddot{x} \sin \varphi=L(\dot{\varphi})=d-s \dot{\varphi} .
\end{aligned}
$$

Using $\tau=\omega t, x=x / x^{*}$, and $z=\varphi / \varphi^{*}$, the dimensionless form of (1) can be derived as,

$$
\begin{aligned}
& \dot{x}=y, \\
& \dot{y}=-a y+b x-c x^{3}+d \dot{w} \sin z+d w^{2} \cos z, \\
& \dot{z}=w, \\
& \dot{w}=0.05 \dot{y} \sin z-100 w+200, \\
& a=\frac{b}{\left(m_{1}+m_{0}\right) \omega}, \\
& \omega=\sqrt{\frac{k_{1}}{m_{1}+m_{0}}}, \\
& b=\frac{k_{1}}{\left(m_{1}+m_{0}\right) \omega^{2}}, \\
& c=\frac{k_{\mathrm{nl}} x^{* 2}}{\left(m_{1}+m_{0}\right) \omega^{2}}, \\
& d=\frac{m_{0} r \varphi^{*}}{\left(m_{1}+m_{0}\right) x^{*}}, \\
& \rho_{2}=\frac{d}{\left(J+r^{2} m_{0}\right) \omega^{2} \varphi^{*}}=100 \text {, } \\
& \delta_{2}=\frac{m_{0} r \varphi^{* 2}}{\left(m_{1}+m_{0}\right) x^{*}}, \\
& \rho_{1}=\frac{r m_{0} x^{*}}{\left(J+r^{2} m_{0}\right) \varphi^{*}}=0.05 \text {, } \\
& \rho_{3}=\frac{s \omega \varphi^{*}}{\left(J+r^{2} m_{0}\right) \omega^{2} \varphi^{*}}=200 \text {. }
\end{aligned}
$$

System (2) shows chaotic oscillations when $a=0.1, b=$ $1, c=2, d=8.473$ with initial conditions $[0.1,0.1,0.1,0.1]$. Figure 2 shows the 3D phase portraits of system (2).

\section{Fractional Order Portal Frame (FOPF)}

In this section we derive the fractional order model of the portal frame (FOPF) from the integer order dimensionless model discussed in (2). There are three commonly used definitions of the fractional order differential operator, namely, Grunwald-Letnikov, Riemann-Liouville, and Caputo [1214]. We use the fractional order system derived from the 


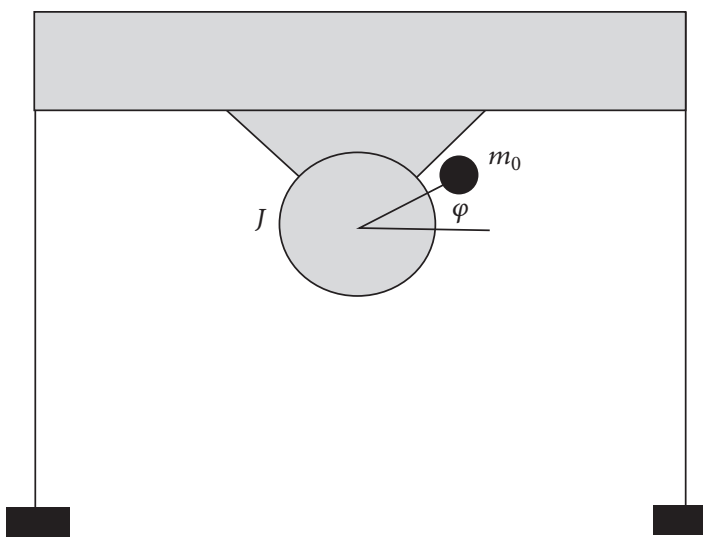

(a)

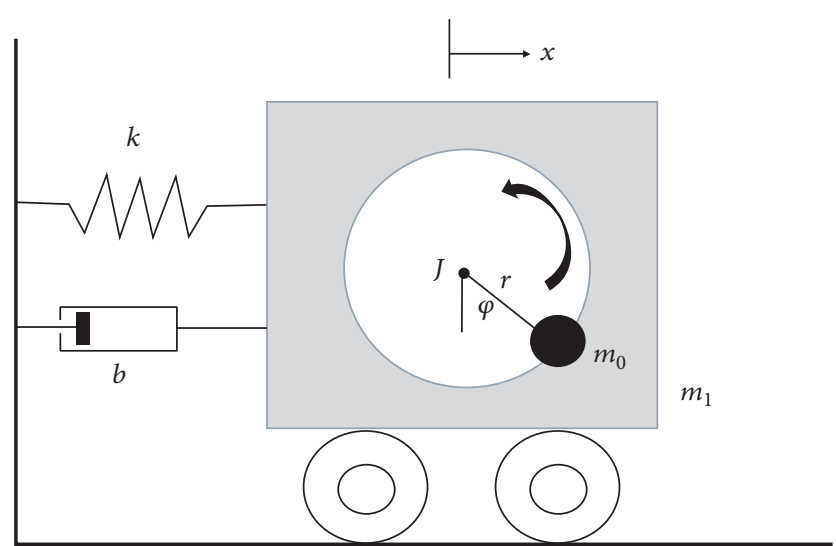

(b)

FIgure 1: (a) Portal frame with nonideal excitation. (b) Coupled oscillator approximation.
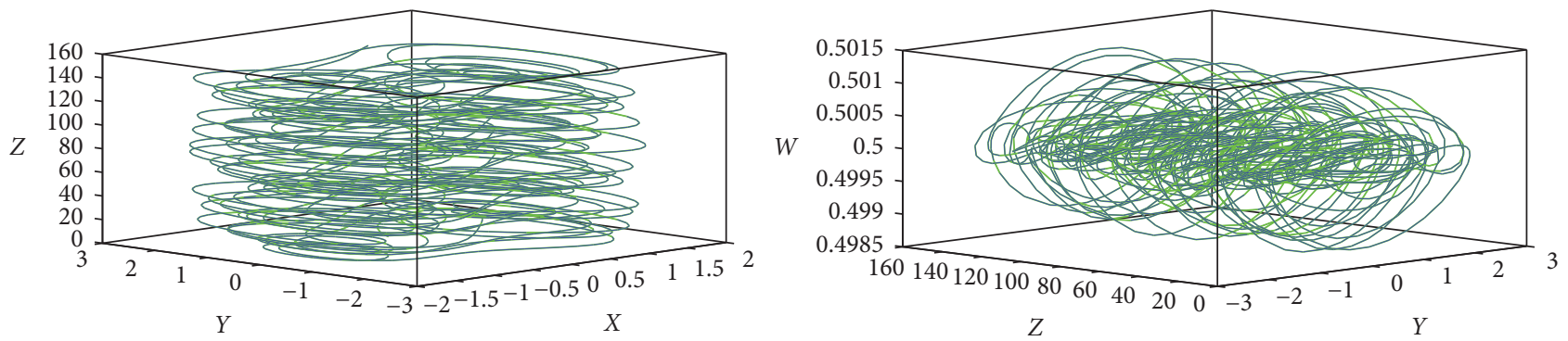

FIGURE 2: 3D phase portraits of the portal frame system (2).

portal frame system (1) with the Grunwald -Letnikov (GL) definition, which is defined as

$$
\begin{aligned}
{ }_{a} D_{t}^{q} f(t) & =\lim _{h \rightarrow 0}\left\{\frac{1}{h^{q}} \sum_{j=0}^{[(t-q) / h]}(-1)^{j}\left(\begin{array}{l}
q \\
j
\end{array}\right) f(t-j h)\right\} \\
& =\lim _{h \rightarrow 0}\left\{\frac{1}{h^{q}} \Delta_{h}^{q} f(t)\right\},
\end{aligned}
$$

where $a$ and $t$ are limits of the fractional order equation, $\Delta_{h}^{q} f(t)$ is generalized difference, $h$ is the step size, and $q$ is the fractional order of the differential equation.

For numerical calculations the above equation is modified as

$$
{ }_{(t-L)} D_{t}^{q} f(t)=\lim _{h \rightarrow 0}\left\{h^{-q} \sum_{j=0}^{N(t)} b_{j} f(t-j h)\right\} .
$$

Theoretically fractional order differential equations use infinite memory. Hence when we want to numerically calculate or simulate the fractional order equations we have to use finite memory principal, where $L$ is the memory length and $h$ is the time sampling.

$$
N(t)=\min \left\{\left[\frac{t}{h}\right],\left[\frac{L}{h}\right]\right\} .
$$

The binomial coefficients required for the numerical simulation are calculated as

$$
b_{j}=\left(1-\frac{a+q}{j}\right) b_{j-1} .
$$

Using the relations (4) and (5), the fractional order portal frame dimensionless model can be derived as

$$
\begin{aligned}
D^{q_{x}} x & =y, \\
D^{q_{y}} y & =-a y+b x-c x^{3}+d\left(D^{q_{w}} w\right) \sin z+d w^{2} \cos z, \\
D^{q_{z}} z & =w, \\
D^{q_{w}} w & =0.05\left(D^{q_{y}} y\right) \sin z-100 w+200 .
\end{aligned}
$$

The parameter values are $a=0.1, b=1, c=2, d=$ 8.473 and the initial conditions are $[0.1,0.1,0.1,0.1]$. The system shows its largest Lyapunov exponent $(0.08138)$ when $q=0.998$ against its integer order Lyapunov exponent of 0.075 [3], confirming that the chaotic oscillations are more in fractional order close to 1 compared to the integer order. Figure 3 shows the 3D phase portraits of the FOPF system.

\section{Dynamic Analysis of FOPF System}

4.1. Bifurcation Analysis with Parameters. The FOPF system described in (8) has four parameters $a, b, c, d$ and to study the 

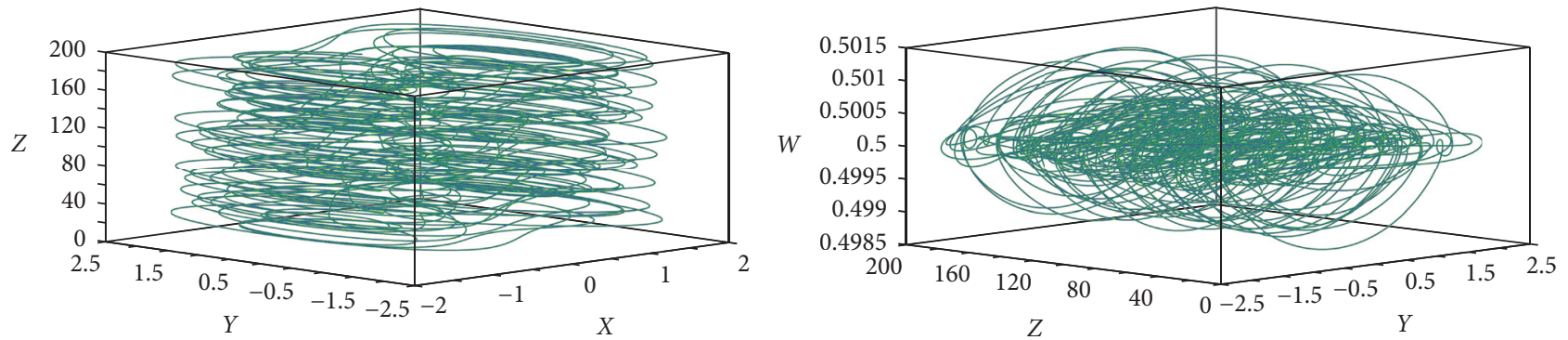

Figure 3: 3D phase portraits of the FOPF system.
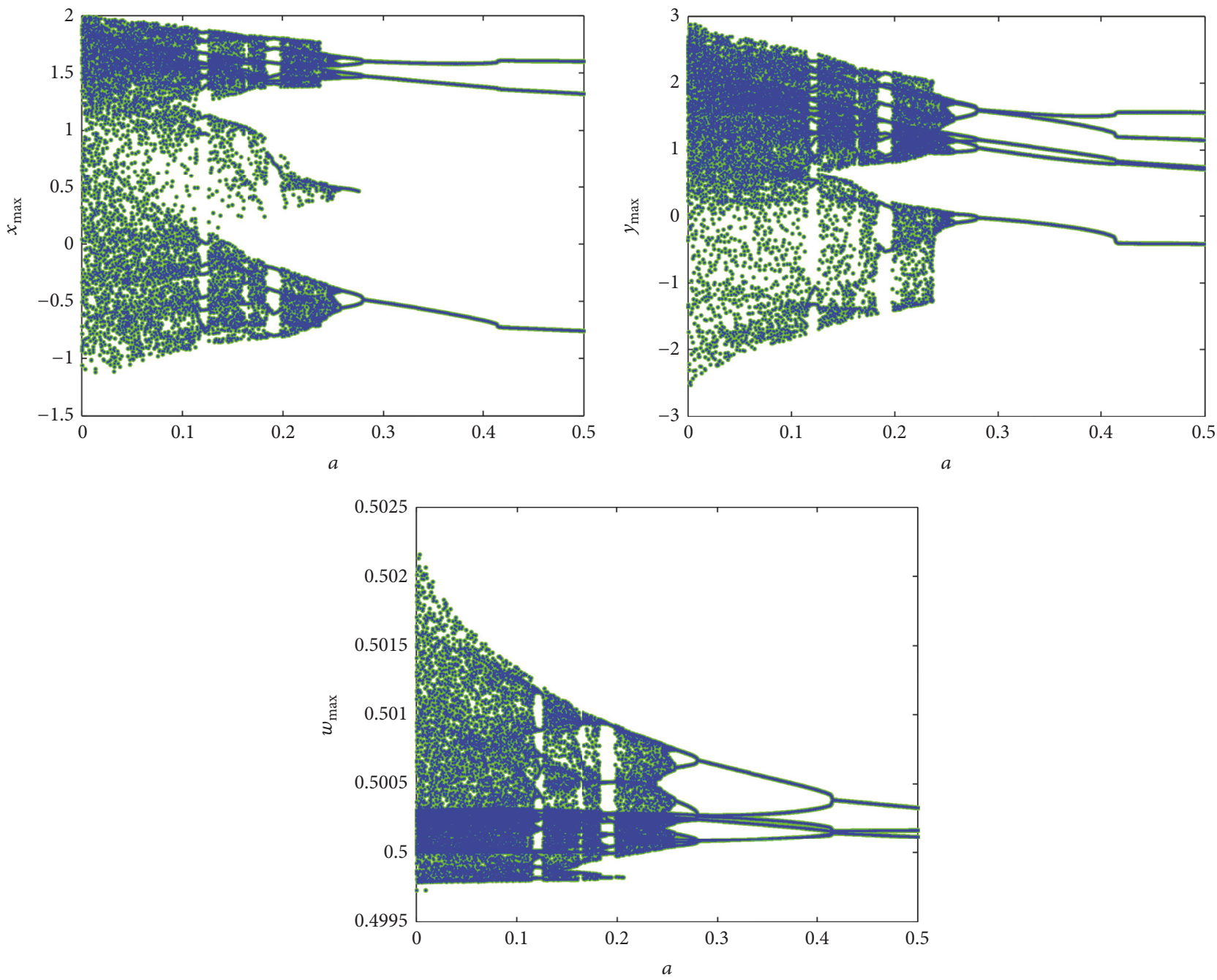

FIGURE 4: Bifurcation of FOPF system with $a$.

impact of these parameters of the FOPF system, we derive the bifurcation plots. Figure 4 shows the bifurcation plots of FOPF system for the parameter $a$. As can be seen from Figure 4, the FOPF system shows multiple chaotic regions for $a$. There exists a wide band chaotic region for $0 \leq a \leq 0.12$ and the systems maximum Lyapunov exponent $(0.08138)$ is shown when $a=0.1$. The system shows quasi-periodic state when positive Lyapunov exponent becomes zero for $0.12 \leq$ $a \leq 0.14$. The second chaotic region of the FOPF system is seen for $0.14 \leq a \leq 0.18$ and the Lyapunov exponent of the system lies between [0.0317, 0.0453]. For a narrow band of $0.18 \leq a \leq 0.19$ the FOPF systems show multiple stable limit cycles attracting the neighboring trajectories exhibiting selfsustained oscillations. There exists the third chaotic region for $0.21 \leq a \leq 0.25$ and the FOPF system takes period halving route for $0.25 \leq a \leq 0.27$ to exit chaotic oscillations.

Figure 5 shows the bifurcation of the FOPF system for $b$. The FOPF system takes a routine period doubling route 

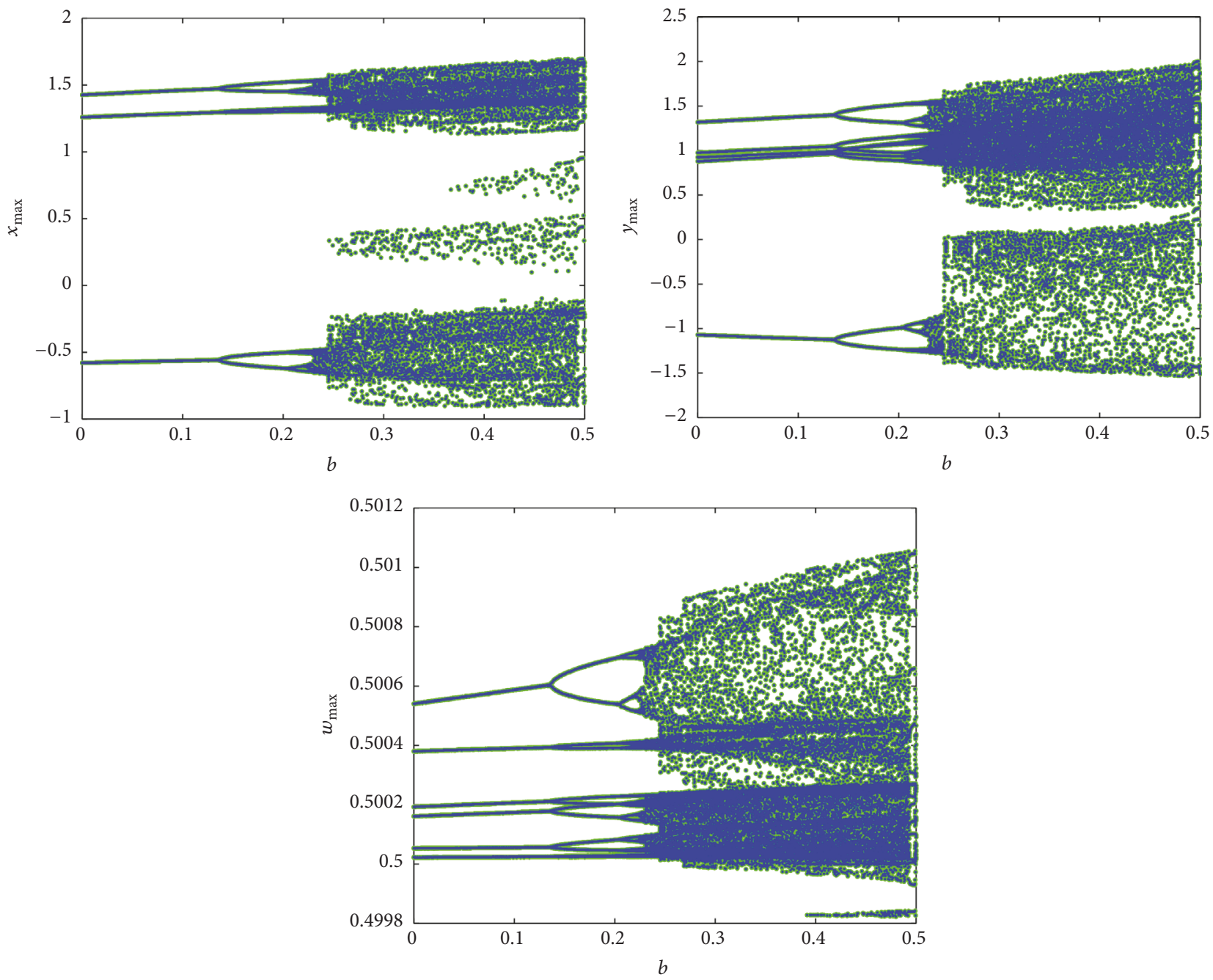

FIGURE 5: Bifurcation plots of FOPF system for $b$.

to chaos and shows multiple period doubling for $0.15 \leq$ $b \leq 0.25$. Figure 6 shows the bifurcation of FOPF system with parameter $c$. The FOPF system shows multiple chaotic regions for $c$. For $0.2 \leq c \leq 0.3$ the FOPF systems show a chaotic region. Stable and unstable limit cycles are seen for $0.41 \leq c \leq 0.63$ and $0.63 \leq c \leq 1.27$, respectively. The system takes a routine period doubling rote to chaos for $1.35 \leq c \leq 1.57$ and $1.63 \leq c \leq 1.94$. A narrow band of quasichaotic state exists for $1.94 \leq c \leq 1.97$ where the systems only positive Lyapunov exponent becomes zero. The third chaotic region is seen for $1.97 \leq c \leq 2.4$ and the systems maximum Lyapunov exponent (0.08138) exists when $c=2$.

Figure 7 shows the bifurcation of FOPF system with $d$. The system enters in to chaos with period doubling for $6.50 \leq$ $d \leq 6.92$. The first chaotic region exists for $6.92 \leq d \leq 7.4$. Then the system enters in to the second chaotic region $(7.6 \leq$ $d \leq 8.37)$ through period doubling $(7.4 \leq d \leq 7.6)$. There exists a small band of quasi-chaotic region for $8.37 \leq d \leq 8.39$ when the Lyapunov exponent goes to zero. The third chaotic region exists for $8.39 \leq d \leq 9$ and the FOPF systems largest Lyapunov exponent $(0.08138)$ is seen when $d=8.473$.

4.2. Bifurcation Analysis with Fractional Order. The bifurcation of the FOPF system with fractional orders is another important topic of investigation. Figure 8 shows the bifurcation plots of the FOPF system for various fractional orders. The FOPF system shows chaotic oscillations for the commensurate orders $q_{i}>0.99$ and the largest positive Lyapunov exponent $(0.08138)$ of the nonideal portal frame system exists in the fractional order $q=0.998$ against the integer order Lyapunov exponent of 0.075 [3]. This clearly confirms that fractional order chaos control is effective compared to the integer order control as discussed in [23].

4.3. Bicoherence. Higher order spectra have been used to study the nonlinear interactions between frequency modes [41]. Let $x(t)$ be a stationary random process defined as

$$
x(t)=\sum_{n=1}^{N} A_{n} e^{j \omega_{n} t}+A_{n}^{*} e^{-j \omega_{n} t},
$$



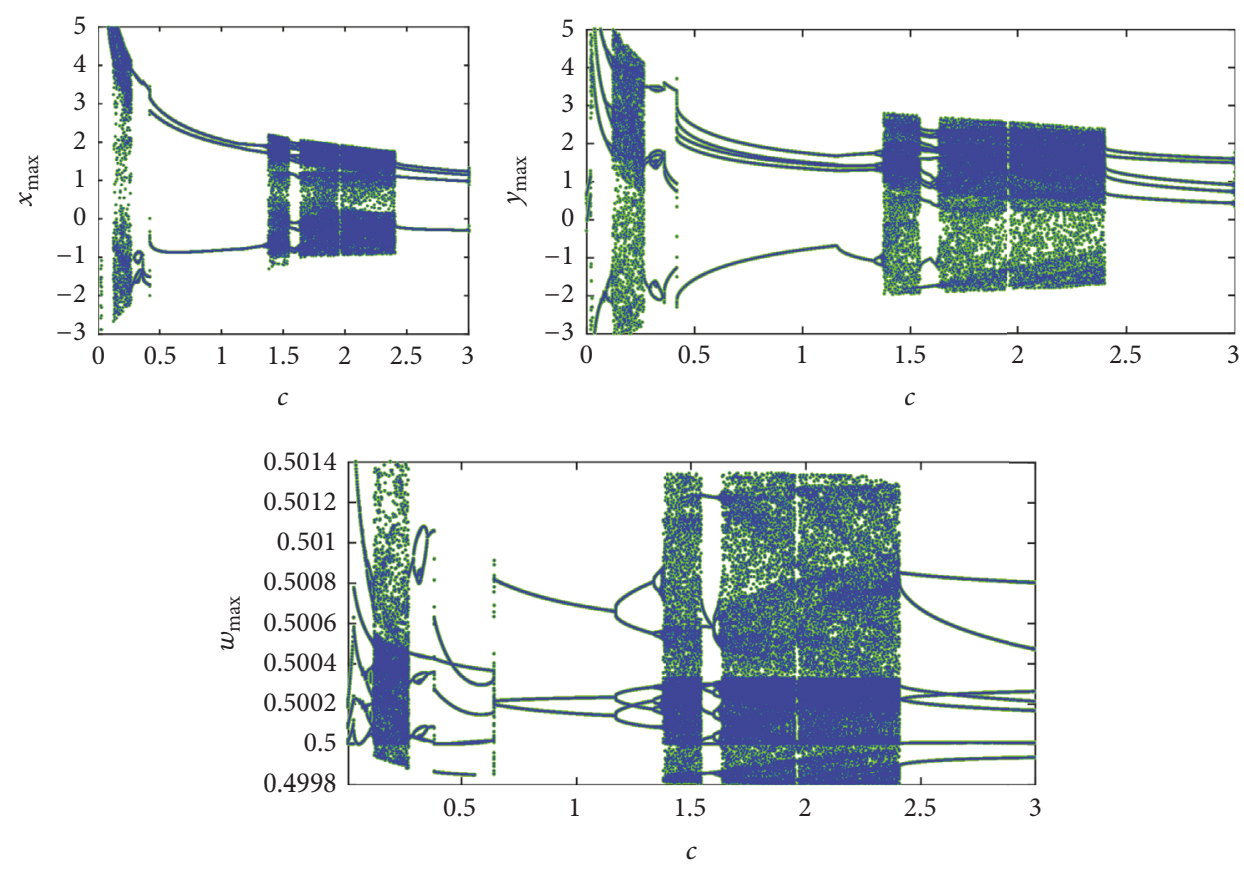

FIGURE 6: Bifurcation of FOPF system for $c$.
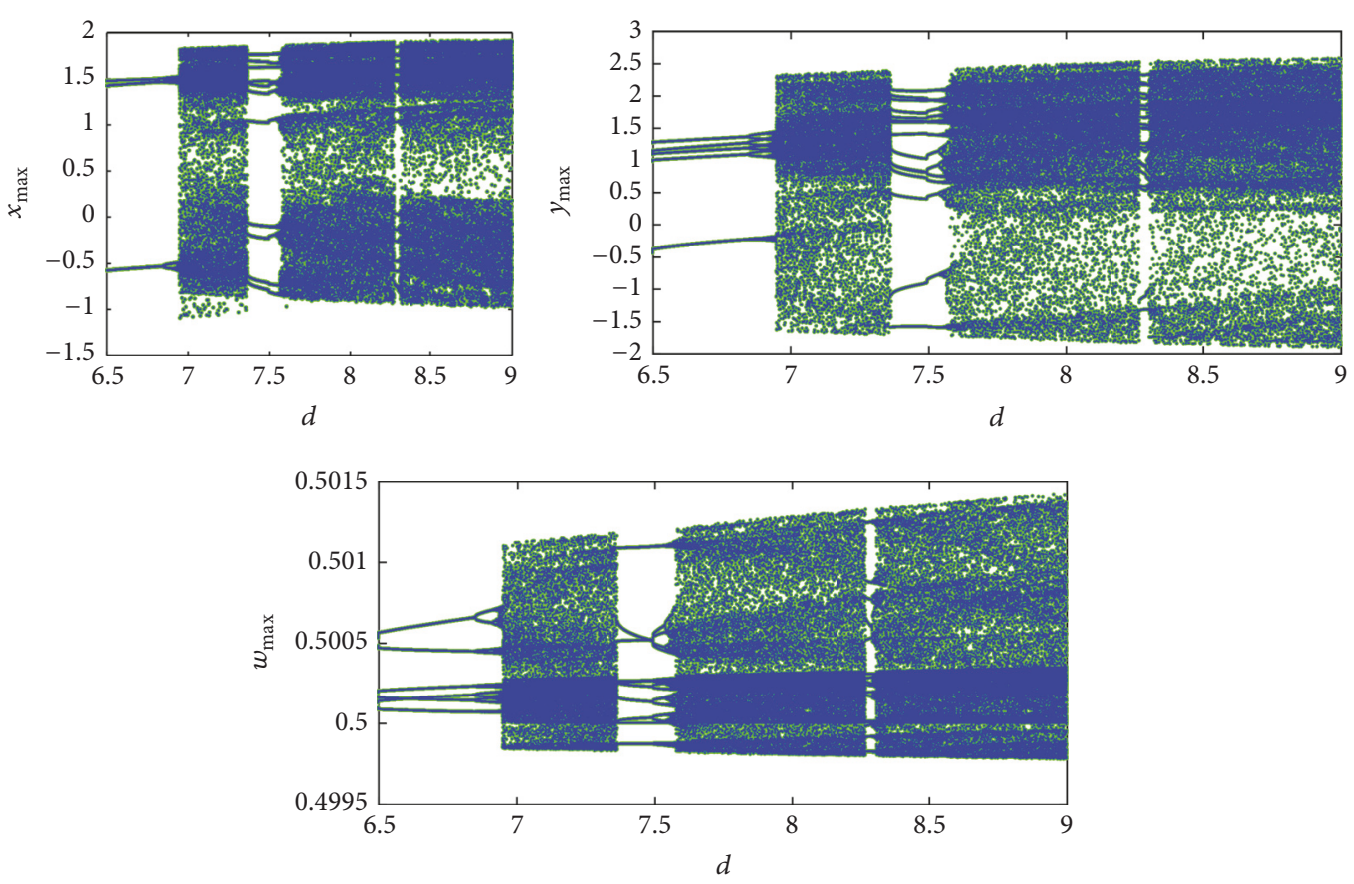

FIGURE 7: Bifurcation of FOPF system with $d$.

where $\omega$ is the angular frequency, $n$ is the frequency modal index, and $A_{n}$ are the complex Fourier coefficients. The power spectrum can be defined as

$$
P\left(\omega_{k}\right)=E\left[A_{\omega_{k}} A_{\omega_{k}}^{*}\right]
$$

and discrete bispectrum can be defined as

$$
B\left(\omega_{k}, \omega_{j}\right)=E\left[A_{\omega_{k}} A_{\omega_{j}} A_{\omega_{k}+\omega_{j}}^{*}\right] .
$$

If the modes are independent then the average triple products of Fourier components are zero resulting in a zero bispectrum [41]. The study of bicoherence is to give an indication of the relative degree of phase coupling between triads of frequency components. The motivation to study the bicoherence is twofold. First, the bicoherence can be used to extract information due to deviations from Gaussianity and suppress additive (colored) Gaussian noise. Second, the 


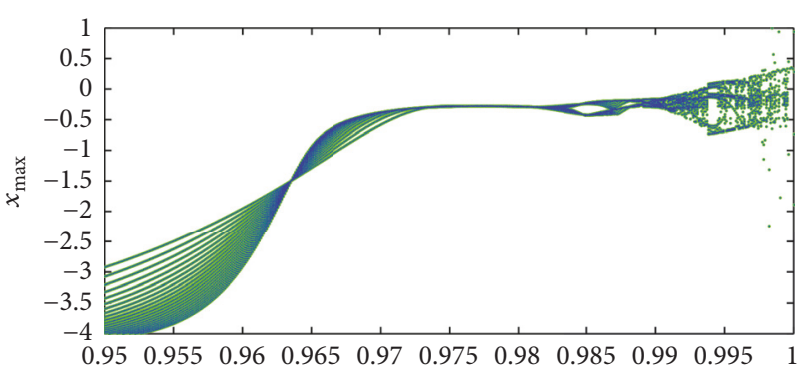

$q$

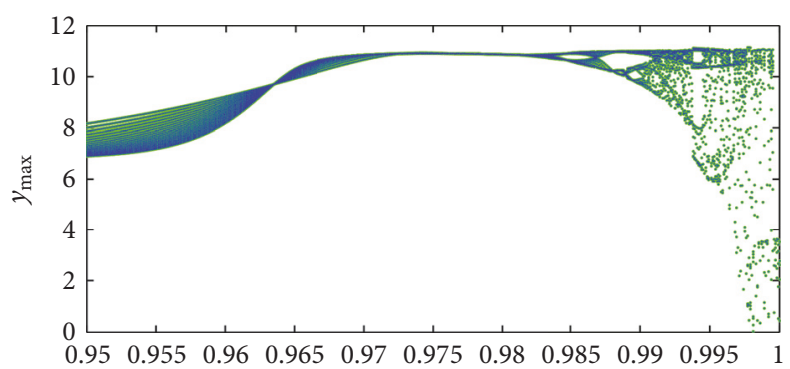

$q$

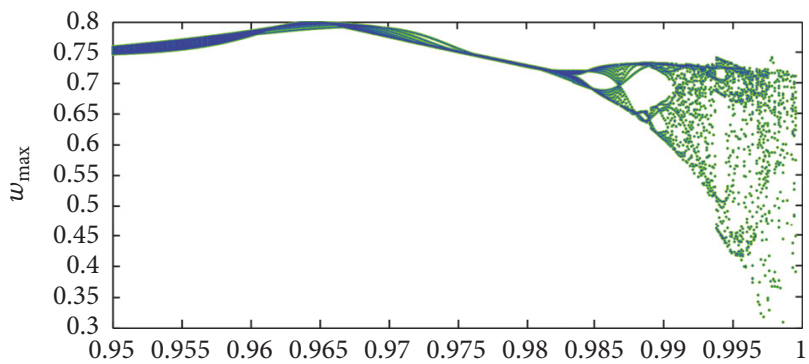

9

FIGURE 8: Bifurcation of FOPF system with fractional order $q$.

bicoherence can be used to detect and characterize asymmetric nonlinearity in signals via quadratic phase coupling or identify systems with quadratic nonlinearity. The bicoherence is the third-order spectrum. Whereas the power spectrum is second-order statistics, formed from $X^{\prime}(f) * X(f)$, where $X(f)$ is the Fourier transform of $x(t)$, the bispectrum is third-order statistics formed from $X\left(f_{j}\right) * X\left(f_{k}\right) * X^{\prime}\left(f_{j}+\right.$ $f_{k}$ ). The bispectrum is therefore a function of a pair of frequencies $\left(f_{j}, f_{k}\right)$. It is also a complex-valued function. The (normalized) square amplitude is called the bicoherence (by analogy with the coherence from the cross-spectrum). The bispectrum is calculated by dividing the time series into $M$ segments of length $N \_$seg, calculating their Fourier transforms and biperiodogram and then averaging over the ensemble. Although the bicoherence is a function of two frequencies the default output of this function is a onedimensional output, the bicoherence refined as a function of only the sum of the two frequencies. The autobispectrum of a chaotic system is given by Pezeshki [42]. He derived the autobispectrum with the Fourier coefficients.

$$
B\left(\omega_{1}, \omega_{2}\right)=E\left[A\left(\omega_{1}\right) A\left(\omega_{2}\right) A^{*}\left(\omega_{1}+\omega_{2}\right)\right],
$$

where $\omega_{n}$ is the radian frequency and $A$ are the Fourier coefficients of the time series. The normalized magnitude spectrum of the bispectrum known as the squared bicoherence is given by

$$
b\left(\omega_{1}, \omega_{2}\right)=\frac{\left|B\left(\omega_{1}, \omega_{2}\right)\right|^{2}}{P\left(\omega_{1}\right) P\left(\omega_{2}\right) P\left(\omega_{1}+\omega_{2}\right)},
$$

where $P\left(\omega_{1}\right)$ and $P\left(\omega_{2}\right)$ are the power spectra at $f_{1}$ and $f_{2}$.

Figures $9(\mathrm{a})$ and 9 (b) show the bicoherence contours of the FOPF system for state $x$ and all states together, respectively. Shades in yellow represent the multifrequency components contributing to the power spectrum. From Figures 9(a) and 9(b) the cross-bicoherence is significantly nonzero and nonconstant, indicating a nonlinear relationship between the states. As can be seen from Figure 9(a), the spectral power is very low as compared to the spectral power of all states together (Figure 9(b)) indicating the existence of multifrequency nodes. Also Figure 9(b) shows the nonlinear coupling (straight lines connecting multiple frequency terms) between the states. The yellow shades/lines and nonsharpness of the peaks, as well as the presence of structure around the origin in figures (cross-bicoherence), indicate that the nonlinearity between the states $x, y, z, w$ is not of the quadratic nonlinearity and hence may be because of nonlinearity of higher dimensions. The most two dominant frequencies $\left(f_{1}, f_{2}\right)$ are taken for deriving the contour of bicoherence. The sampling frequency $\left(f_{s}\right)$ is taken as the reference frequency. Direct FFT is used to derive the power spectrum for individual frequencies and Hankel operator is used as the frequency mask. Hanning window is used as the FIR filter to separate the frequencies [40].

\section{Fractional Order Adaptive Sliding Mode Control (FOASMC)}

In this section we derive the fractional order adaptive sliding mode controllers for suppressing the chaotic oscillations in the FOPF system. As discussed in [3], it is sufficient to control states $x$ and $y$; hence we include two controllers $u_{x}$ and $u_{y}$. To include uncertainties, we assume the system parameters $(a, b, c, d)$ are unknown. We redefine the FOPF system with the FOASMC controllers as

$$
\begin{aligned}
& \dot{x}=y+u_{x}, \\
& \dot{y}=-a y+b x-c x^{3}+d \dot{w} \sin z+d w^{2} \cos z+u_{y},
\end{aligned}
$$



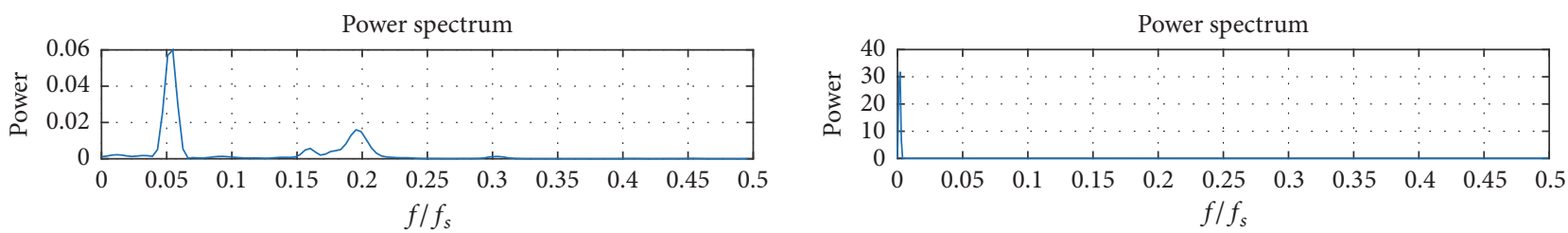

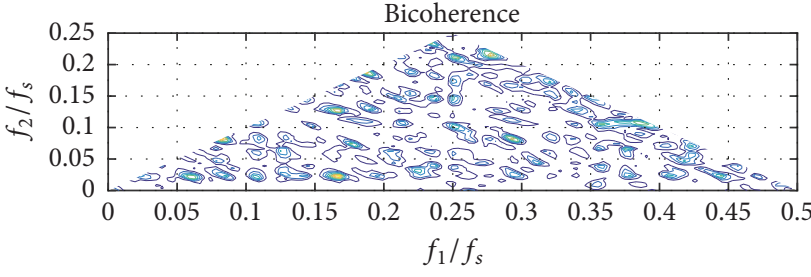

(a)

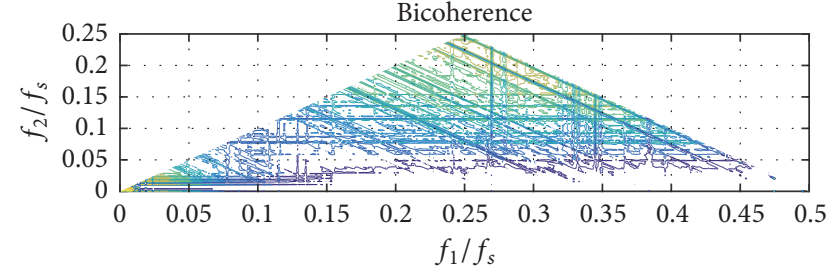

(b)

FIgUrE 9: (a) Bicoherence of state $x$. (b) Bicoherence of all states of FOPF system.

$$
\begin{aligned}
\dot{z} & =w, \\
\dot{w} & =0.05 \dot{y} \sin z-100 w+200,
\end{aligned}
$$

where $u_{i}$ is the adaptive sliding mode controller with $i=x, y$ The sliding surfaces $[39,40,43]$ are defined as

$$
\begin{aligned}
& s_{x}=x+k_{x} \int_{0}^{t} x(\tau) d \tau, \\
& s_{y}=y+k_{y} \int_{0}^{t} y(\tau) d \tau .
\end{aligned}
$$

The Fractional derivative of sliding surfaces (15) is given by

$$
\begin{aligned}
& D^{q_{x}} s_{x}=D^{q_{x}} x+k_{x} x, \\
& D^{q_{y}} s_{y}=D^{q_{y}} y+k_{y} y .
\end{aligned}
$$

To include uncertainties in the FOPF system, the parameters $a, b, c, d$ are assumed unknown and hence the parameter estimation errors are defined as

$$
\begin{aligned}
& e_{a}=\widehat{a}-a, \\
& e_{b}=\widehat{b}-b, \\
& e_{c}=\widehat{c}-c, \\
& e_{d}=\widehat{d}-d .
\end{aligned}
$$

The fractional derivative of the parameter estimation errors (17) is

$$
\begin{aligned}
& D^{q_{y}} e_{a}=D^{q_{y}} \widehat{a}, \\
& D^{q_{y}} e_{b}=D^{q_{y}} \widehat{b}, \\
& D^{q_{y}} e_{c}=D^{q_{y}} \widehat{c}, \\
& D^{q_{y}} e_{d}=D^{q_{y}} \widehat{d} .
\end{aligned}
$$

We define the Lyapunov candidate function

$$
V=\frac{1}{2}\left[s_{x}^{2}+s_{y}^{2}+e_{a}^{2}+e_{b}^{2}+e_{c}^{2}+e_{d}^{2}\right] .
$$

The first derivative of (19) is

$$
\begin{aligned}
\dot{V}= & s_{x} \cdot \dot{s}_{x}+s_{y} \cdot \dot{s}_{y}+e_{a} \cdot \dot{e}_{a}+e_{b} \cdot \dot{e}_{b}+e_{c} \cdot \dot{e}_{c}+e_{d} \\
& \cdot \dot{e}_{d}
\end{aligned}
$$

By definition of fractional calculus [12-14],

$$
\dot{x}(t)=D_{t}^{1-q} \cdot D_{t}^{q} x(t) .
$$

Using (21) in (20),

$$
\begin{aligned}
\dot{V}= & s_{i} \cdot D_{t}^{1-q} \cdot D_{t}^{q} s_{i}-(b-\widehat{b})\left(D_{t}^{1-q} \cdot D_{t}^{q} \widehat{b}\right) \\
& -(a-\widehat{a})\left(D_{t}^{1-q} \cdot D_{t}^{q} \widehat{a}\right)-(c-\widehat{c})\left(D_{t}^{1-q} \cdot D_{t}^{q} \widehat{c}\right) \\
& -(d-\widehat{d})\left(D_{t}^{1-q} \cdot D_{t}^{q} \widehat{d}\right),
\end{aligned}
$$

where $i=x, y$ and $q$ is the commensurate order of the system. Finding the sign of the Lyapunov first derivative using (22) seems difficult and hence we use the modified fractional order Lyapunov method defined by Rajagopal et al. [39, 40, 44] as

$$
\frac{1}{2} D_{t}^{q} x^{2}(t) \leq x(t) \frac{1}{2} D_{t}^{q} x(t), \quad q \in(0,1) .
$$

Using (14), (16), (18), and (23) in (20)

$$
\begin{aligned}
\dot{V} & \leq s_{x}\left[y+u_{x}+k_{x} x\right]+s_{y}\left[-a y+b x-c x^{3}\right. \\
& \left.+d \dot{w} \sin z+d w^{2} \cos z+u_{y}+k_{y} y\right]+e_{a} D^{q_{y}} \widehat{a} \\
& +e_{b} D^{q_{y}} \widehat{b}+e_{c} D^{q_{y}} \widehat{c}+e_{d} D^{q_{y}} \widehat{d} .
\end{aligned}
$$

The adaptive sliding mode controllers are defined as

$$
\begin{aligned}
u_{x}= & -y-k_{x} x-\eta_{x} \operatorname{sgn}\left(s_{x}\right)-\rho_{x} s_{x}, \\
u_{y}= & \widehat{a} y-\widehat{b} x+\widehat{c} x^{3}-\widehat{d} \dot{w} \sin z-\widehat{d} w^{2} \cos z-k_{y} y \\
& \quad-\eta_{y} \operatorname{sgn}\left(s_{y}\right)-\rho_{y} s_{y},
\end{aligned}
$$




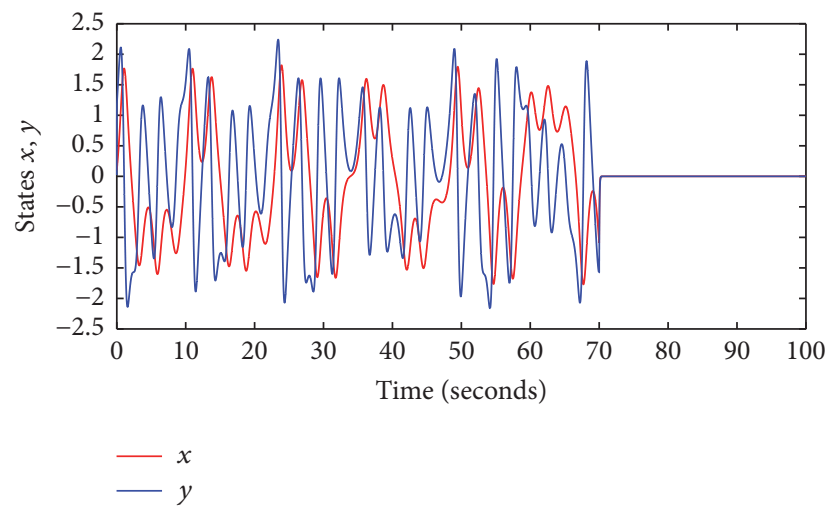

FIgURE 10: Time history of FOPF states (controller in action at $t=$ $70 \mathrm{~s})$.

where $\eta_{i}, \rho_{i}$, and $k_{i}$ are positive constants for $i=x, y$. The parameter estimation laws are derived as

$$
\begin{aligned}
& D^{q_{y} \widehat{a}}=-s_{y} y, \\
& D^{q_{y} \widehat{b}}=s_{y} x, \\
& D^{q_{y} \widehat{c}}=s_{y} x^{3}, \\
& D^{q_{y}} \widehat{d}=s_{y}\left[w^{2} \cos z+\dot{w} \sin z\right] .
\end{aligned}
$$

Using (25) and (26) in (24), we simplify the Lyapunov candidate function as

$$
\dot{V} \leq-\eta_{x}\left|s_{x}\right|-\eta_{y}\left|s_{y}\right|-\rho_{x} s_{x}^{2}-\rho_{y} s_{y}^{2}
$$

as $\eta_{i}$ and $\rho_{i}$ are all positive for $i=x, y ; \dot{V}$ is negative definite. Using Barbalat's lemma [45], we conclude that $e_{i}(t) \rightarrow 0$ as $t \rightarrow \infty$. Figures 10 and 11 show the time history of the states $(x, y)$ and parameter estimates, respectively. The controller is switched on at time $t=70 \mathrm{~s}$ and the initial conditions of the FOPF system are taken as $[0.1,0.02,0.3,0.04]$ and parameter estimates as $[1,4,5,6]$.

\section{FPGA Implementation of the FOPF System}

For numerically simulating the FOPF control scheme, we first implement the FOPF model in FPGA $[39,40]$ using the Xilinx (Vivado) System Generator toolbox in Simulink. Firstly we configure the available built in blocks of the System Generator toolbox. The Add/Sub blocks are configured with zero latency and $32 / 16$ bit fixed point settings. The output of the block is configured to rounded quantization in order to reduce the bit latency. For the multiplier block a latency of 1 is configured and the other settings are same as in Add/Sub block. Next we will have to design the fractional order integrator which is not a readily available block in the System Generator $[39,40]$. Hence we implement the integrators using the mathematical relation discussed in Section 3 and the value of $h$ is taken as 0.001 and the initial conditions are fed in to the forward register with fractional order taken as $q=0.998$ for FOPF system. Figure 12 shows the Xilinx RTL schematics of the

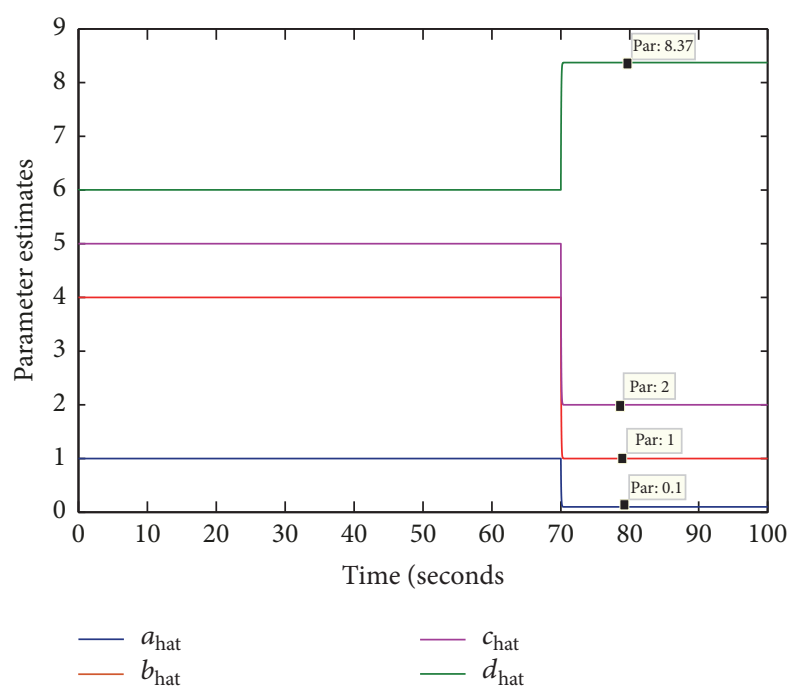

FIGURE 11: Time history of FOPF parameter estimates (controller in action at $t=70 \mathrm{~s})$.

FOPF system implemented in Kintex 7 (Device $=7 \mathrm{k} 160 \mathrm{t}$ Package $=\mathrm{fbg} 484 \mathrm{~S}$ ) and Figure 13 shows the 3D phase portraits of the FOPF system implemented in FPGA. Here we used a sampling period of $0.01 \mathrm{~s}$. Increasing the sampling time period in some implementation may lead to a clock frequency mismatch and hence plays a critical role in implementation. Also negative time slack may also create problems while implementing the design and hence choosing constraints may also be critical in cases where the number of logical operations is more. Avoiding DDR clocks help in reducing the route delays. Table 1 shows the resources consumed and for analyzing the power consumed by the controllers, we use the approximation methodology discussed in [39, 40]. Figures 14(a) and 14(b) show the power utilization for fractional order $q=0.998$ and power utilization for various fractional orders. It confirms that larger power will be consumed by the system when the FOPF system shows the largest Lyapunov exponents (FOPF $q=0.998$ ) as shown in Figure 14(b).

6.1. FPGA Implementation of FOASMC Synchronisation. For real-time implementation of the proposed control scheme, the FOASMC controller can be implemented in FPGA and the output voltages from the respective control pins can be configured with an active magnetorheological damper. In this section we implement the proposed fractional order adaptive sliding mode controllers (FOASMC) derived in (23) along with the fractional parameter update laws (24) and sliding surfaces (13). For implementation of the entire control scheme we use Virtex 7 (Device: xc7vx980t-1ffg1926). The fractional order of the FOPF master system, slave system, and the FOASMC is kept as $q=0.998$. For analyzing the power consumed by the controllers, we use the approximation methodology discussed in [43]. It confirms that larger power will be consumed by the controller when the master and the slave system show largest Lyapunov exponents. Figure 15 shows the RTL schematics of the fractional order ASMC 
TABLE 1: Resources utilized by the FOPF system.

\begin{tabular}{|c|c|c|c|c|c|}
\hline \multirow{2}{*}{ Kintex 7 k160t } & \multirow{2}{*}{ Utilization } & \multirow{2}{*}{ Available } & \multirow{2}{*}{ Utilization $\%$} & \multicolumn{2}{|c|}{ Clock frequency } \\
\hline & & & & $f_{\max }$ & Used \\
\hline LUT & 974 & 101400 & 0.96 & $500 \mathrm{Mhz}$ & $214 \mathrm{Mhz}$ \\
\hline FF & 850 & 202800 & 0.42 & $300 \mathrm{Mhz}$ & $132 \mathrm{Mhz}$ \\
\hline DSP & 36 & 600 & 6.00 & $500 \mathrm{Mhz}$ & $244 \mathrm{Mhz}$ \\
\hline $\mathrm{IO}$ & 129 & 285 & 45.26 & $250 \mathrm{Mhz}$ & $129 \mathrm{Mhz}$ \\
\hline BUFG & 1 & 32 & 3.13 & $300 \mathrm{Mhz}$ & $112 \mathrm{Mhz}$ \\
\hline
\end{tabular}

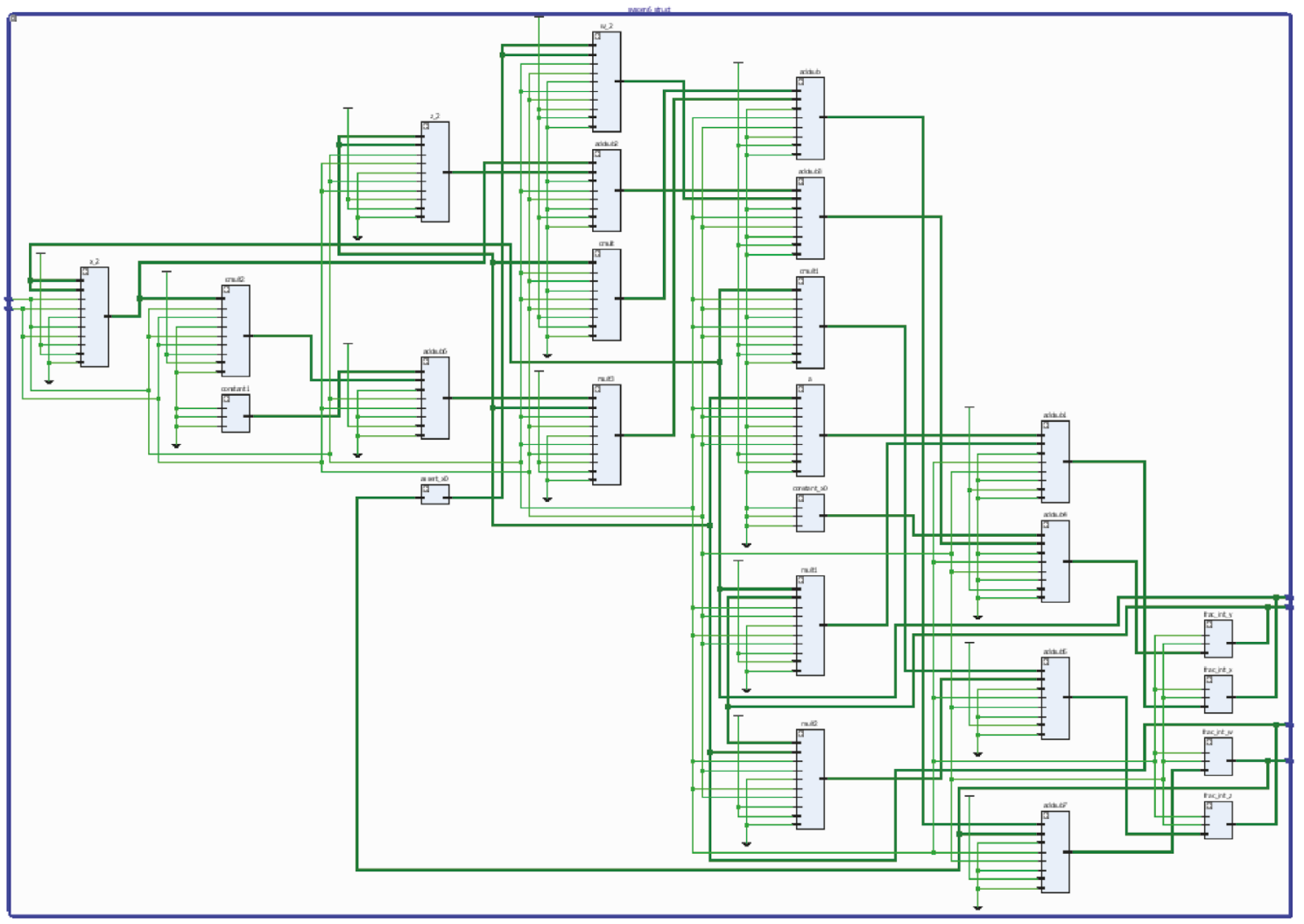

Figure 12: Xilinx RTL schematics of the FOPF system implemented in Kintex 7.
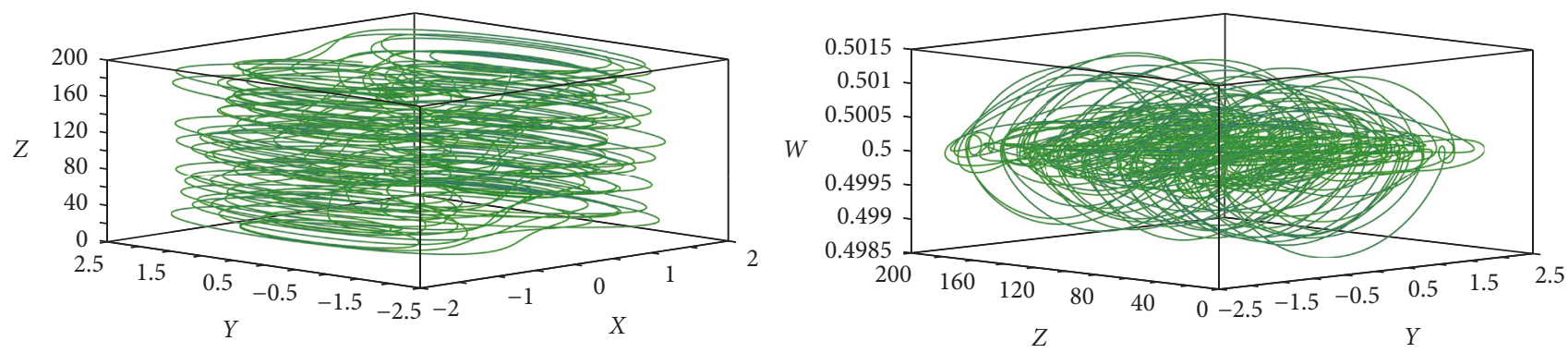

FIGURE 13: 3D phase portraits of the FOPF system implemented in FPGA with order $q=0.998$.

controllers implemented in Virtex 7 (Device: xc7vx980t1ffg1926). Figures 16(a) and 16(b) show the power utilization of the controller and power utilization with change in fractional orders, respectively. Figures 17 and 18 show the controlled states and estimated parameters of the FOPF system, respectively. To utilize the power of FPGA, the computation needs to be divided into several independent blocks of threads that can be executed simultaneously. The 


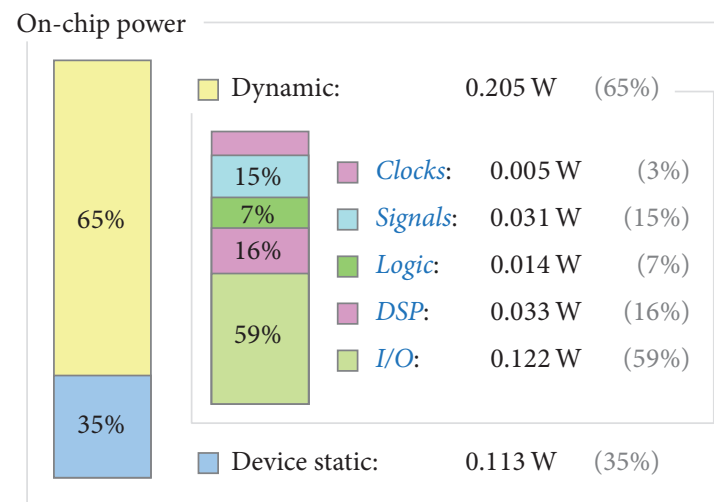

(a)

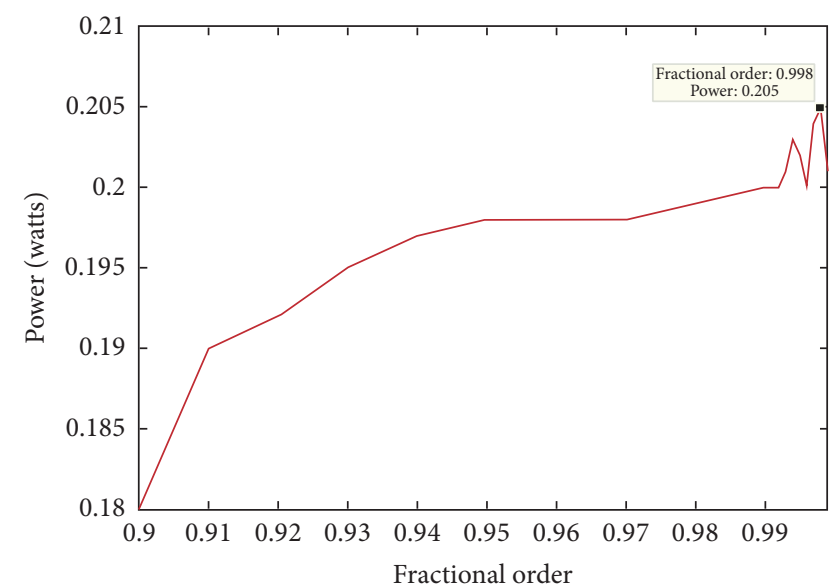

(b)

FIGURE 14: (a) Power utilized and (b) power utilization versus fractional order of FOPF.

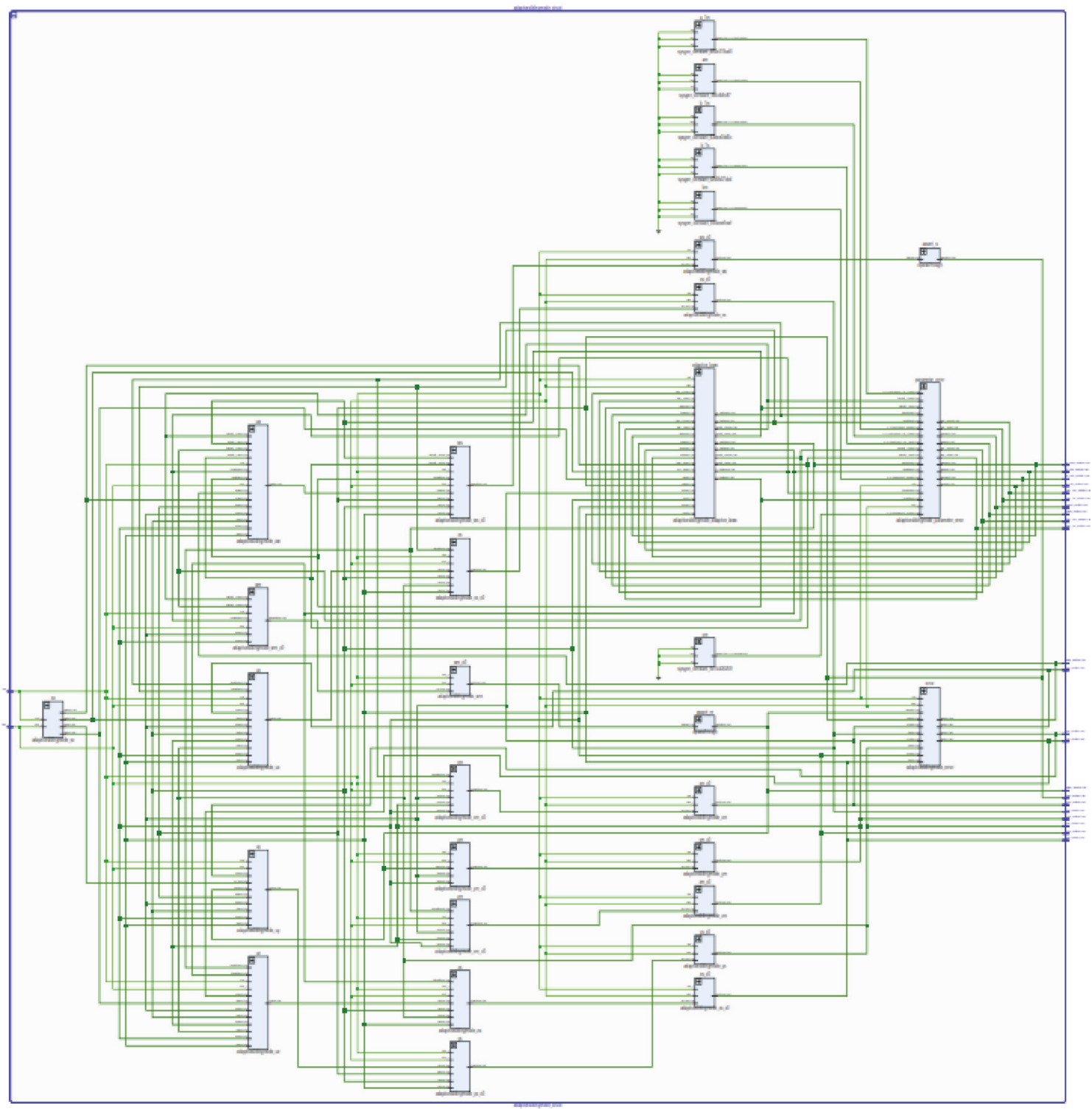

FIgURE 15: Xilinx RTL schematics of the FOASMC controllers implemented in Virtex 7. 


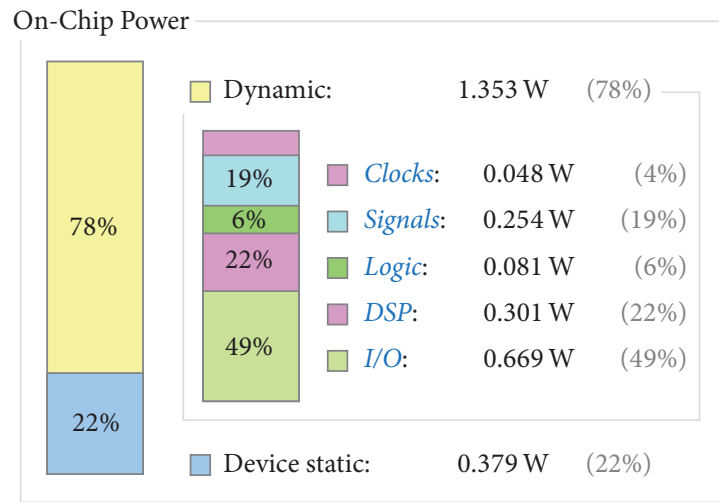

(a)

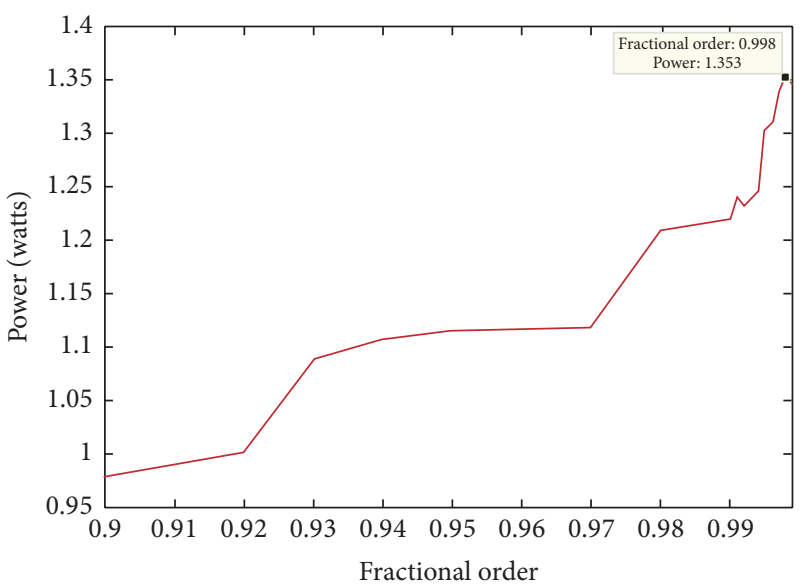

(b)

FIgURE 16: (a) Power utilized and (b) power utilization versus fractional order of FOASMC.

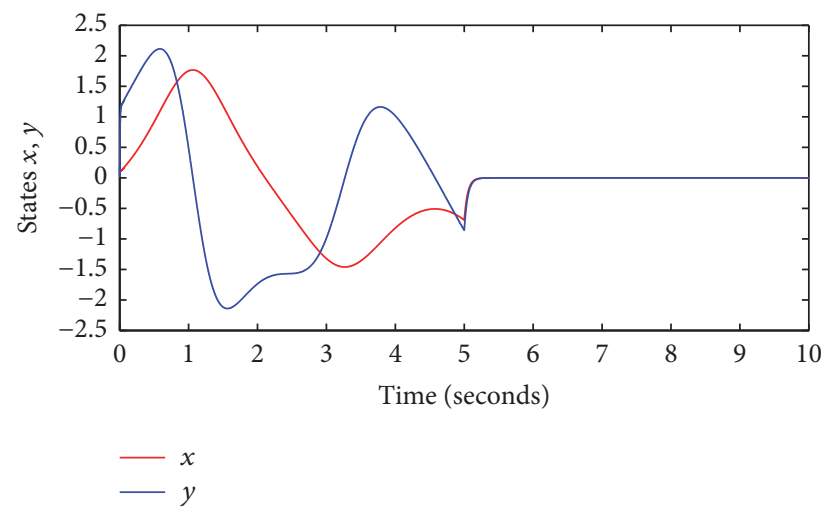

FIGURE 17: Controlled states of FOPF system (controller in action at $t=5 \mathrm{~s})$.

performance on FPGA is directly related to the number of threads and its performances decrease when number of branching instructions increases. The fractional order operators are implemented as building blocks and the socalled "frame delay" is not noticeable in the FPGA hardware implementation due to its parallel data structure, unlike a microprocessor-based implementation. While FPGA implementation has a reputation for being difficult to design, with the help of systematic methodology, a system can be put together with less work than is required for more traditional software-based realizations.

\section{Conclusion}

Most of the literatures have investigated chaotic oscillations in an integer order portal frame with nonideal loading. In this paper we investigated the chaotic oscillations of a fractional order portal frame. Existence of chaotic oscillations in a fractional order portal frame is investigated with a positive Lyapunov exponent. Bifurcation plots in the parameter space are investigated for the regions of chaotic oscillations.

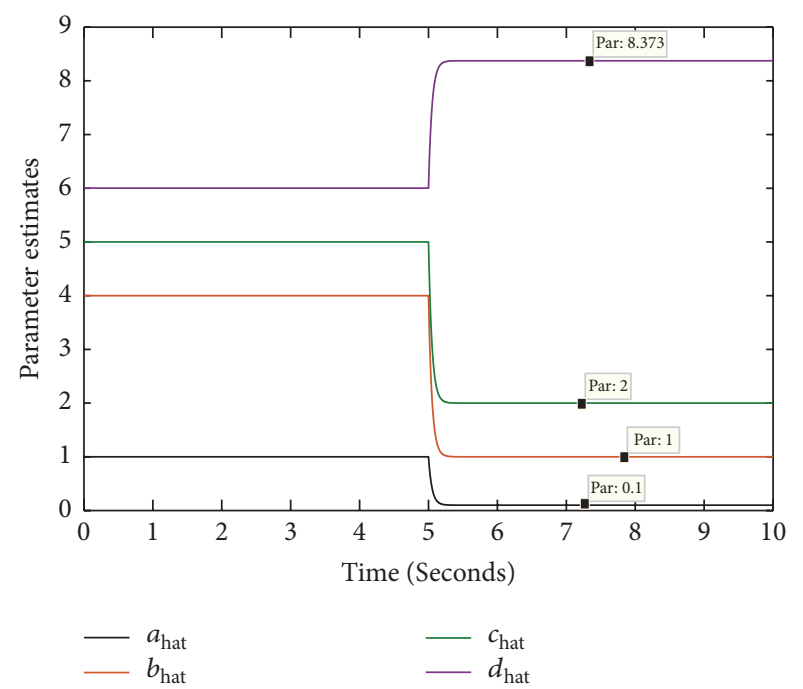

FIgURE 18: Estimated parameters of FOPF system (controller in action at $t=5 \mathrm{~s}$ ).

Investigation of bicoherence contours shows the quadratic nonlinearities and its existence because of autocorrelation and cross correlation. To control the chaotic oscillations an adaptive sliding mode control scheme is derived and numerically verified. As can be seen from Figures 9 and 10, the proposed control scheme is effective even in the presence of uncertainties in the parameters. For real-time implementation, the fractional order portal frame system with the adaptive sliding mode control algorithm is implemented in FPGA and as can be seen from Figures 16 and 17, the FPGA implemented controllers are effective in chaos suppression. For real-time chaos suppression, the output voltages from the FPGA pins can be connected to MR dampers.

\section{Conflicts of Interest}

The authors declare that there are no conflicts of interest regarding the publication of this paper. 


\section{References}

[1] K. A. Castaõ, L. C. S. Goes, and J. M. Balthazar, "A note on the attenuation of the sommerfeld effect of a non-ideal system taking into account a MR damper and the complete model of a DC motor," Journal of Vibration and Control, vol. 17, no. 7, pp. 1112-1118, 2010.

[2] A. A. Nanha Djanan, B. R. Nana Nbendjo, and P. Woafo, "Electromechanical control of vibration on a plate submitted to a non-ideal excitation," Mechanics Research Communications, vol. 54, pp. 72-82, 2013.

[3] A. M. Tusset, V. Piccirillo, J. M. Balthazar, and R. M. L. Rebello Da Fonseca Brasil, "On suppression of chaotic motions of a portal frame structure under non-ideal loading using a magneto-rheological damper," Journal of Theoretical and Applied Mechanics, vol. 53, no. 3, pp. 653-664, 2015.

[4] A. D. S. Barr and D. C. McWhannell, "Parametric instability in structures under support motion," Journal of Sound and Vibration, vol. 14, no. 4, pp. 491-509, 1971.

[5] M. L. R. M. Brasil and C. E. N. Mazzilli, "A general FEM formulation of nonlinear dynamics applied to accessing the statical loading effect upon the dynamic response of planar frames," Applied Mechanics Reviews, vol. 46, no. 11, pp. 110-117, 1993.

[6] R. M. L. R. F. Brasil and J. M. Balthazar, Chaotic Motions of a Simple Portal Frame, 2000, http://www.abcm.org.br/ anais/conem/2000/NC9196.pdf.

[7] M. Rafikov, J. M. Balthazar, and Â. M. Tusset, "An optimal linear control design for nonlinear systems," Journal of the Brazilian Society of Mechanical Sciences and Engineering, vol. 30, no. 4, pp. 279-284, 2008.

[8] R. M. L. R. F. Brasil and J. M. Balthazar, "Nonlinear oscillations of a portal frame structure excited by a nonideal motor," in Proceedings of the 2000 2nd International Conference Control of Oscillations and Chaos Proceedings, vol. 2, pp. 275-278, St. Petersburg, Russia, July 2000.

[9] J. M. Balthazar, R. M. L. F. Brasil, J. L. P. Felix et al., "Dynamics behaviour of an elastic non-ideal (NIS) portal frame, including fractional nonlinearities," Journal of Physics: Conference Series, vol. 721, no. 1, Article ID 12004, 2016.

[10] J. L. Palacios Felix, J. M. Balthazar, and R. M. L. R. F. Brasil, "On saturation control of a non-ideal vibrating portal frame foundation type shear-building," Journal of Vibration and Control, vol. 11, no. 1, pp. 121-136, 2005.

[11] I. Iliuk, J. Balthazar, A. Tusset et al., "A non-ideal portal frame energy harvester controlled using a pendulum," The European Physical Journal Special Topics, vol. 222, no. 7, pp. 1575-1586, 2013.

[12] D. Baleanu, K. Diethelm, E. Scalas, and J. J. Trujillo, Fractional Calculus: Models and Numerical Methods, World Scientific, Singapore, 2014.

[13] Y. Zhou, Basic Theory of Fractional Differential Equations, World Scientific, Singapore, 2014.

[14] K. Diethelm, The Analysis of Fractional Differential Equations, vol. 2004 of Lecture Notes in Mathematics, Springer, Berlin, Germany, 2010.

[15] M. Pourmahmood Aghababa, "Robust finite-time stabilization of fractional-order chaotic systems based on fractional lyapunov stability theory," Journal of Computational and Nonlinear Dynamics, vol. 7, no. 2, Article ID 021010, pp. 21-31, 2012.

[16] E. A. Boroujeni and H. R. Momeni, "Non-fragile nonlinear fractional order observer design for a class of nonlinear fractional order systems," Signal Processing, vol. 92, no. 10, pp. 2365-2370, 2012.

[17] Z. Ruoxun and J. Gong, "Synchronization of the fractionalorder chaotic system via adaptive observer," Systems Science \& Control Engineering, vol. 2, no. 1, pp. 751-754, 2014.

[18] I. Petras, "Methods for simulation of the fractional order chaotic systems," Acta montanastica Slovaca, vol. 11, no. 4, pp. 273-277, 2006.

[19] W. Trzaska Zdzislaw, "Matlab Solutions of Chaotic Fractional Order Circuits," Intech Open, 2013, https://cdn.intechopen.com/ pdfs-wm/21404.pdf.

[20] L.-L. Li, G.-Y. Gu, and L.-M. Zhu, "A fractional-order active damping control approach for piezo-actuated nanopositioning stages," in Proceedings of the 1st International Conference on Manipulation, Automation and Robotics at Small Scales (MARSS '16), pp. 1-6, Paris, France, July 2016.

[21] A. Chevalier, C. Copot, D. Copot, C. M. Ionescu, and R. De Keyser, "Fractional-order feedback control of a poorly damped system," in Proceedings of the 19th IEEE International Conference on Automation, Quality and Testing, Robotics (AQTR '14), pp. 14, Cluj-Napoca, Romania, May 2014.

[22] C. I. Muresan, E. H. Dulf, and O. Prodan, "A fractional order controller for seismic mitigation of structures equipped with viscoelastic mass dampers," Journal of Vibration and Control, vol. 22, no. 8, pp. 1980-1992, 2016.

[23] H. Sheng, Y. Q. Chen, and T. S. Qiu, "Optimal Fractional-Order Damping Strategies," in Fractional Processes and FractionalOrder Signal Processing: Techniques and Applications, Springer London, London, UK, 2011.

[24] Y. Li, Y. Chen, and I. Podlubny, "Stability of fractional-order nonlinear dynamic systems: Lyapunov direct method and generalized Mittag-Leffler stability," Computers \& Mathematics with Applications, vol. 59, no. 5, pp. 1810-1821, 2010.

[25] J. A. Gallegos and M. A. Duarte-Mermoud, "On the Lyapunov theory for fractional order systems," Applied Mathematics and Computation, vol. 287/288, pp. 161-170, 2016.

[26] M. S. Tavazoei and M. Haeri, "Chaos control via a simple fractional-order controller," Physics Letters A, vol. 372, no. 6, pp. 798-807, 2008.

[27] Y. Gao and K. T. Chau, "Hopf bifurcation and chaos in synchronous reluctance motor drives," IEEE Transactions on Energy Conversion, vol. 19, no. 2, pp. 296-302, 2004.

[28] G. Revel, A. E. Leon, D. M. Alonso, and J. L. Moiola, "Multiparameter bifurcation analysis of subsynchronous interactions in DFIG-based wind farms," Electric Power Systems Research, vol. 140, pp. 643-652, 2016.

[29] A. Zarei and S. Tavakoli, "Hopf bifurcation analysis and ultimate bound estimation of a new 4-D quadratic autonomous hyperchaotic system," Applied Mathematics and Computation, vol. 291, pp. 323-339, 2016.

[30] Y. A. Kuznetsov, Elements of Applied Bifurcation Theory, vol. 112 of Applied Mathematical Sciences, Springer, Berlin, Germany, 1995.

[31] N. Jabli, H. Khammari, M. F. Mimouni, and R. Dhifaoui, "Bifurcation and chaos phenomena appearing in induction motor under variation of PI controller parameters," WSEAS Transactions on Systems, vol. 9, no. 7, pp. 784-793, 2010.

[32] E. Tlelo-Cuautle, A. D. Pano-Azucena, J. J. Rangel-Magdaleno, V. H. Carbajal-Gomez, and G. Rodriguez-Gomez, "Generating a 50 -scroll chaotic attractor at $66 \mathrm{MHz}$ by using FPGAs," Nonlinear Dynamics, vol. 85, no. 4, pp. 2143-2157, 2016. 
[33] Q. Wang, S. Yu, C. Li et al., "Theoretical design and FPGA-based implementation of higher-dimensional digital chaotic systems," IEEE Transactions on Circuits and Systems I: Regular Papers, vol. 63, no. 3, pp. 401-412, 2016.

[34] E. Dong, Z. Liang, S. Du, and Z. Chen, “Topological horseshoe analysis on a four-wing chaotic attractor and its FPGA implement," Nonlinear Dynamics, vol. 83, no. 1-2, pp. 623-630, 2016.

[35] E. Tlelo-Cuautle, V. H. Carbajal-Gomez, P. J. Obeso-Rodelo, J. J. Rangel-Magdaleno, and J. C. Núñez-Pérez, "FPGA realization of a chaotic communication system applied to image processing," Nonlinear Dynamics, vol. 82, no. 4, pp. 1879-1892, 2015.

[36] V. Rashtchi and M. Nourazar, "FPGA implementation of a realtime weak signal detector using a duffing oscillator," Circuits, Systems, and Signal Processing, vol. 34, no. 10, pp. 3101-3119, 2015.

[37] E. Tlelo-Cuautle, J. J. Rangel-Magdaleno, A. D. Pano-Azucena, P. J. Obeso-Rodelo, and J. C. Nunez-Perez, "FPGA realization of multi-scroll chaotic oscillators," Communications in Nonlinear Science and Numerical Simulation, vol. 27, no. 1-3, pp. 66-80, 2015.

[38] Y.-M. Xu, L.-D. Wang, and S.-K. Duan, "A memristor-based chaotic system and its field programmable gate array implementation," Acta Physica Sinica, vol. 65, no. 12, Article ID 120503, 2016.

[39] K. Rajagopal, A. Karthikeyan, and A. K. Srinivasan, "FPGA implementation of novel fractional-order chaotic systems with two equilibriums and no equilibrium and its adaptive sliding mode synchronization," Nonlinear Dynamics, vol. 87, no. 4, pp. 2281-2304, 2017.

[40] K. Rajagopal, L. Guessas, S. Vaidyanathan, A. Karthikeyan, and A. Srinivasan, "Dynamical analysis and FPGA implementation of a novel hyperchaotic system and its synchronization using adaptive sliding mode control and genetically optimized PID Control," Mathematical Problems in Engineering, vol. 2017, Article ID 7307452, 14 pages, 2017.

[41] D. M. W. Leenaerts, "Higher-order spectral analysis to detect power-frequency mechanisms in a driven Chua's circuit," International Journal of Bifurcation and Chaos, vol. 7, no. 6, pp. 14311440, 1997.

[42] C. Pezeshki, "Bispectral analysis of possessing chaotic motion," Journal of Sound and Vibration, vol. 137, no. 3, pp. 357-368, 1990.

[43] C. Wang, R. Chu, and J. Ma, "Controlling a chaotic resonator by means of dynamic track control," Complexity, vol. 21, no. 1, pp. 370-378, 2015.

[44] K. Rajagopal, S. Vaidhyanathan, and A. Karthikeyan, "Dynamic analysis and chaos suppression in a fractional order brushless DC motor," Electrical Engineering, vol. 99, no. 2, pp. 721-733, 2017.

[45] H. K. Khalil, Nonlinear Systems, Pearson Education, New York, NY, USA, 3rd edition, 2002. 


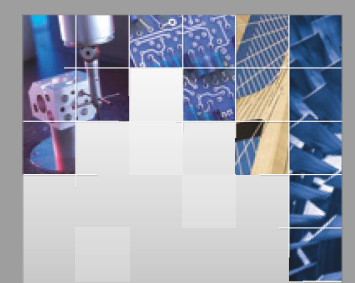

\section{Enfincering}
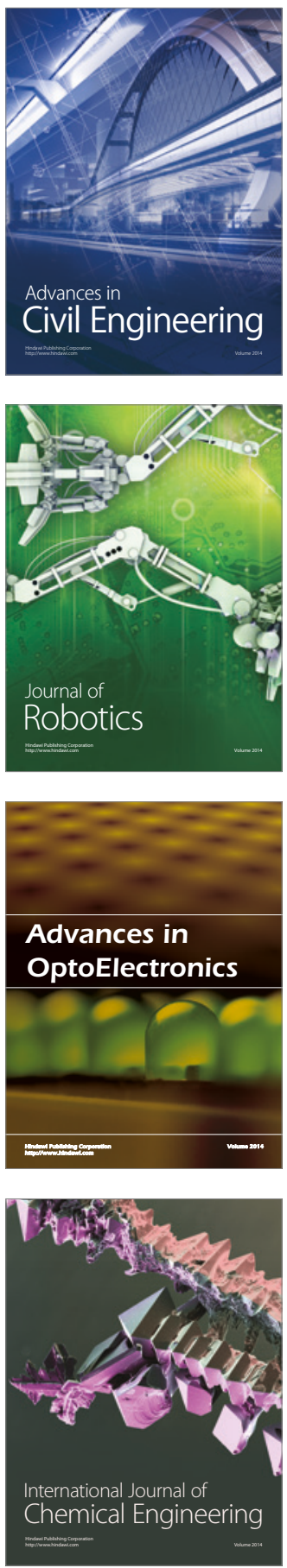

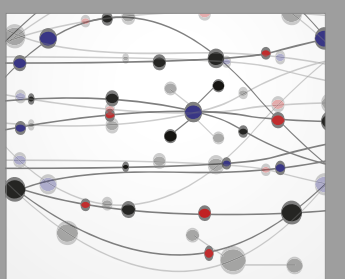

The Scientific World Journal

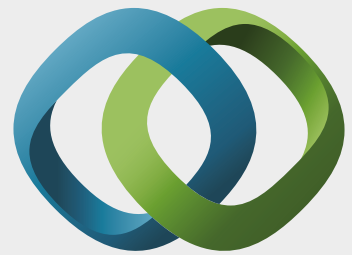

\section{Hindawi}

Submit your manuscripts at

https://www.hindawi.com
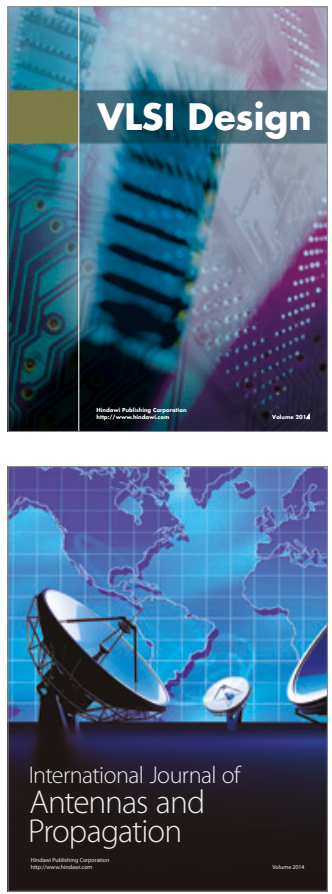

\section{Rotating}

Machinery
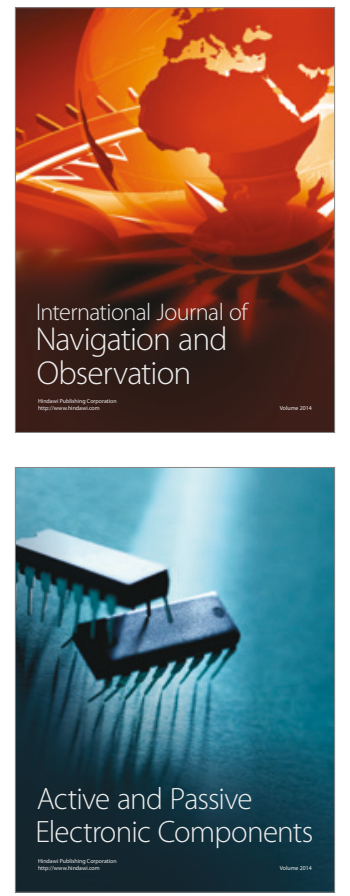
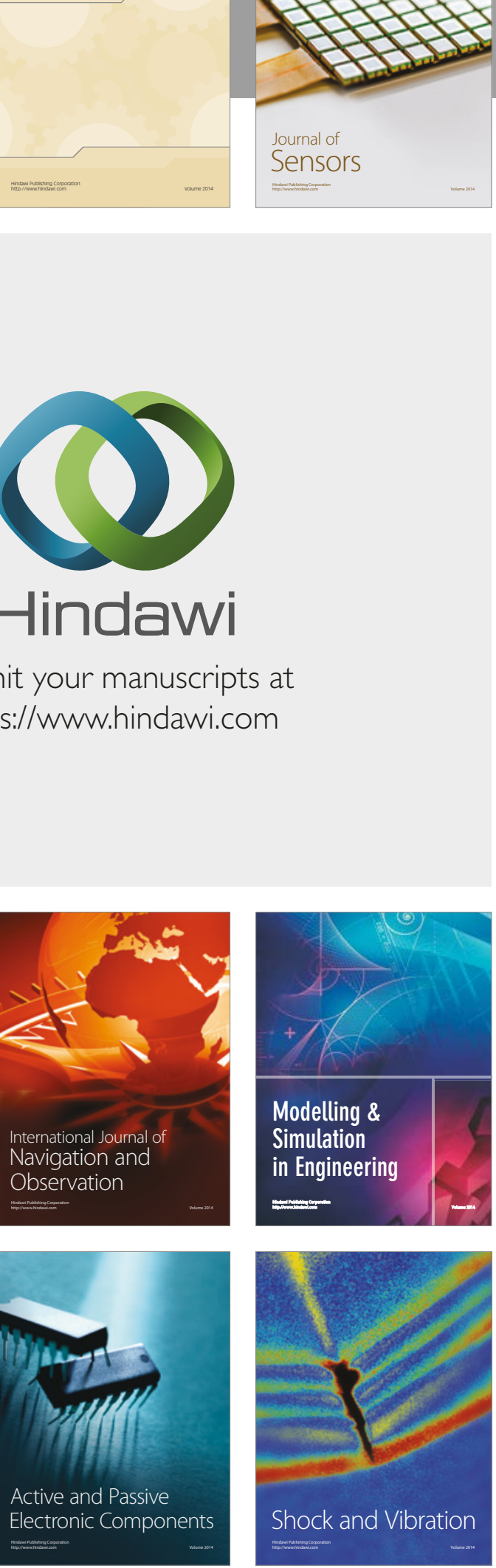
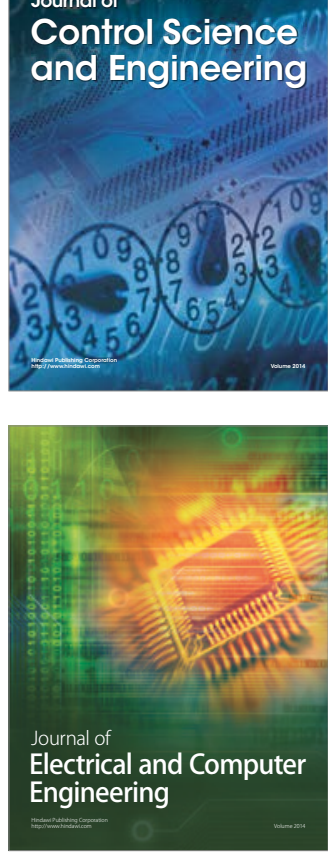

Distributed

Journal of

Control Science

and Engineering
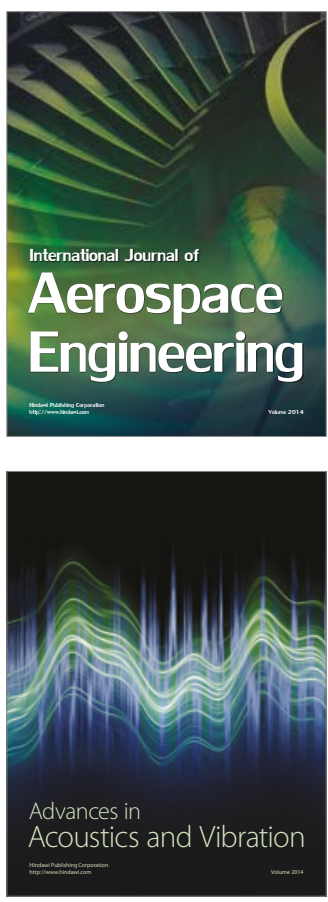

Sensor Networks 